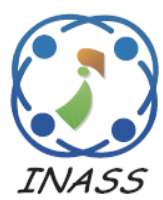

\title{
Efficient Segmentation of Lung Using FIDRM Filter and Successive Peak Quantization Transform
}

\author{
Mohamed Kasim Jannathl Firdouse ${ }^{1^{*}}$ \\ Mururgan Balasubramanian ${ }^{2}$ \\ ${ }^{1}$ Research \& Development Centre, Bharathiar University, India \\ ${ }^{2}$ Department of Computer Science \& Engineering, Annamalai University, India \\ * Corresponding author's Email: jfirdouse@ gmail.com
}

\begin{abstract}
Computed Tomography (CT) is the main source for analyzing the lung diseases and the preplanning of pulmonary surgeries. The accurate interpretation of chest CT scan makes a challenge to radiologists due to its complex visual nature. This paper proposes a novel method to segment the left and right lungs from the background of CT images to assist the pathologists for the easy and accurate diagnosis of pulmonary diseases. The segmentation of lung is a challenging task due to the anatomical difference of different people. This paper develops a novel algorithm namely successive peak based quantization transform (SPQT) to segment the lungs from the background. This method extracts 8 bit patterns from the input lung image and at last a BYTE data which is formed from 8 bit patterns is produced. Prior to SPQT lung segmentation, the lung image which may contain impulse noise is reduced into noise free one by using 'Fuzzy Impulse Detection and Reduction Method' (FIDRM). The novel lung image hikes the accuracy to a significant level than the existing algorithms.
\end{abstract}

Keywords: SPQT, FIDRM, Morphology operations, Lung segmentation, Noise reduction.

\section{Introduction}

Lung cancer is the leading causes of death in the world. This is due to the reasons like smoking, radon, asbestos and air pollution. Early diagnosis and detection are important for the survival. If it is curable, lung neoplasia is asymptomatic. This makes the diagnosis procedure in the advanced state and due to this the survival rate is becoming low. This point out the screening trials of high risk patients in order to reduce the mortality rate. Screening based on radiographs does not make the reduction in the mortality rate. Because of this low dose computed tomography images are applicable with more efficiently. According to the world health organization (WHO), lung cancer is a spiteful tumour, which is one of the five important threats to human life and the health like disease of heart head blood vessel, malignant tumour, respiratory diseases, suicide and accidents. Lung cancer is the highest occurrence of malignant tumour. Its mortality has increased year by year. Patients have no symptoms in the early stages, so it can be neglected without any experiments. When patients have cough, chest pain and other clinical symptoms then the chest CT images are collected and experiments are done but, still it is difficult to find the tumour. Early lung cancer exists as a pulmonary nodule. So, detecting the pulmonary nodules earlier can cause the diagnosis of lung cancer and the further treatments. In image processing, segmentation refers the method of partitioning a digital image into multiple set of pixels. The main aim of segmentation is to change the illustration of an image into simple and meaningful part which is easier to handle and analyze the problem. Image segmentation is mainly used to locate the boundaries. This results in set of regions that entirely covers the original image. Each pixel in the region is same as the computed property such as color, intensity or texture [1]. Several approaches have been introduced in order to detect and evaluate the lung cancer [2 - 4]. These include the detection of lung cancer with small nodules, segmentation of lung regions including fissures and 
lobes. The efficient information is provided by the pulmonary fissures since they are considered as the physical boundaries between the lobes.

The method in [5] segmented 3Dimensional CT lung image in three steps: 1 . Image denoising based on partial differential equation 2. 3 Dimensional CT image binarization using adaptive thresholding and 3. Lung segmentation by applying the morphological operations. Two methods such as Fourier analysis [6], to assign the probability value corresponding to the goodness of the image quality and three supervised learning methods from machine learning [7] namely, Naive Bayes, Support vector machine and Random forests to classify 4 Dimensional CT into either good or bad based on known training data are incorporated to identify 4 Dimensional CT quality measures. Advantages of this method are: segmenting 4 Dimensional CT data, producing an array of numeric data, providing user friendly analysis tools and effective quality measures of 4 Dimensional CT data. Advanced features like surface imaging manipulations, motion registration, internal and external relationships [8], [9] are the drawbacks of this method. The paper described in [10] proposed a novel computer aided detection system based on a hierarchical vector quantization (VQ) scheme, this provided a more accurate segmentation of lungs from the chest volume. Identification of Initial Nodule candidates(INC) is done with the help of low level VQs. False positive reduction is achieved via rule based filtering operations in conjunction with the feature based support vector machine(SVM) classifier. Image segmentation [11], [12], [13] is achieved by applying self-adaptive VQ algorithm. Two fold cross validation was done for the estimation of the capabilities of the SVM classifier. This could be applied for the detection of juxtapleural nodules. The features incorporated with SVM classifier is used by more sophisticated features such as lung nodule detection and texture features for nodule malignancy diagnosis [14]. The detection rate was relatively high is the main advantage of this method. The drawbacks are, if the method is too stringent, the sensitivity of the system will be reduced. Too many FPs will render to follow the SVM classification, if the method is too unfastened. In the method [15], the random walk and graph cut methods are integrated in order to solve the problems in the lung segmentation. The random walk is considered as the initialization tool for the provision of object seeds to the graph cut segmentation on PET and CT images. A graph with two sub graphs is constructed to keep one for the PET images and the other one for the CT images. A novel energy representation including downhill cost and 3D derivative cost for PET images and a shape penalty cost which is integrated in to the energy function are fully utilized. The final tumour segmentation is done by applying the hierarchical clustering algorithm. The detailed information of the lung structure could not be taken from PET and CT images. The location and the size of the organs are not contained in the structure information. Because of this structure information the identification of the foreground seeds are not facilitated and produced the ambiguity

This work is motivated by Song's method [16] in which the labelling of Markov Random Field (MRF) is formulated on the graph on PET and CT images. The main advantage of this method is its good performance in capturing the fuzzy boundary of the tumour on PET images. SUV distribution feature make the highest performance rate on homogeneous and heterogeneous tumours. This method works on Boykov's graph cut method [17 21]. The drawback is that, this method is not suitable for the noisy environment.

The method in [22] models the stack of chest CT scans in connection with 3D Markov-Gibbs Random Field of voxel wise lungs and CT image intensities. $3 \mathrm{D}$ chest scans are pre-processed by region growing and connected components to identify the background voxels. These are then co-aligned by using B-Spline based non rigid registration [23]. This analyses the original input and its Gaussian Scale Space formed by convolving 3D moving Gaussian kernel [24]. The main advantage is its higher accuracy in segmenting the left and right lungs. The healthy tissues can be incorporated to done this method in the near future as advancement.

The main drawback is that this method is not suitable for the large scale images. The paper [25] uses the texture measures and artificial neural network in order to segment the 3D fissures. The geometric model described by $\mathrm{Pu}$ et al. [26] gives the fissure after applying the smoothing operator. The rate of sensitivity, specificity and coefficient of similarity are high are the benefits of this method. Only the fissures are segmented by this method and it can be extended to segment the lobes also. The display of incredulous 3D lung features for heavily affected patient lung images are the main drawback of his method. As described in paper [27] proposed a convolutional method for identifying the interstitial lung diseases (ILD) by evaluating the convolutional neural network. This involves ILD pattern classification based on ROI or VOI depends on $2 \mathrm{D}$ or $3 \mathrm{D}$ lung images. The $2 \mathrm{D}$ textures described are first gray level statistics, gray level co- 
occurrence matrices, run length matrices and fractal analysis $[28,29]$. The large number of parameters and slow training vectors which aligns the fluctuation with the results is the main drawback of this method. As an advancement, 3D images from multiple detector Computed Tomography (MDCT) to be taken for the further classification. This method [30] extracts the fissure by applying the derivative of stick filter for the fissure enhancement and the post processing of lobe segmentation is carried out. The merits are typical abnormalities including thickened fissure, orientation deviations and the step deformation are well preserved with a distinct non-linear derivatives combination and shape description. The application of fissure detection algorithm could be taken as the estimation [31] of fissure integrity. The severely affected lungs yields the false result is considered as the drawback of this method. The future work is to merge the magnitudinal and directional field responses in order to improve the cloud point object detection based on the DoS filter.

The method [32] incorporates 2D convolutional network in which the results are used for the final classification. The two stage processes are: 1 . Candidate detection and 2. False positive reduction. Three candidate detectors such as solid, sub solid and large solid nodules are used. This increases the detection sensitivity of nodules. The combination of these three nodules in the system detects the nodule [33 - 35]. The method is not robust against the noisy environment and due to that some false detection of fissures can be expected. The fast and fully automatic scheme based on iterative weighted averaging and adaptive curvature threshold is proposed in [36] to facilitate the lung segmentation accurately in order for the inclusion of juxta-pleural nodules and pulmonary vessels. This also ensures the smoothness of the lung boundary. The segmentation scheme consists of four stages namely, image pre-processing, thorax extraction, lung identification and lung contour correction. This method produces more than five segments for the severely affected patient's lung images which is not applicable for the real time application. The preoperative assessment of a patient undergoing anatomic lung resection described in [37] segments the lungs in an efficient manner. Computed the 3D margin measurements, identifies the tumour characteristics such as size and segmental location. This is useful in the pre-operative treatment planning [38]. The method described in [39] has the advantage of speed, less segmentation error and easy implementation as explained by [40]. LBF uses the local segmentation with more iteration and high computation time. This uses the signed distance function with region based active contour model.

The existing methods are suffered by lack of accuracy and more time consuming in lung segmentation. So a new method is required to segment the lungs with higher accuracy. This paper proposes a new method to increase the accuracy of segmentation of lung with the support of fuzzy impulse noise detection and reduction method which suppresses the impulse noise to solve the noisy problems when the time of lung segmentation. A new segmentation method successive peak quantization transform is applied on the noise free images to segment the lung image which is much helpful for the preplanning of surgery. The proposed algorithm is even applicable under the noisy environment and the algorithm complexity is less compared to the existing methods.

The section 2 describes about the working methodology of the FIDRM noise filter and the SPQT lung segmentation method to have a better understanding about the methodologies. The section 3 makes an assessment about the proposed lung segmentation against the two recent research papers. The proposed method uses the fuzzy impulse detection and reduction method which is consistent with the existing fuzzy clustering method. This method is more reliable compared with the performance of the existing methods. The performance of the proposed and the existing works are analysed and tables and charts are drawn. The section 4 concludes the performance improvement of the proposed method. Section V lists about the referred papers which are used for this research.

\section{Proposed method}

This paper introduces a novel method for lung segmentation.

The proposed methods in this research continues with three major modules and are listed below:

- FIDRM based noise reduction

- SPQT based lung segmentation

- Morphology based refinement

Here the noise reduction is performed through the first module and the second module partially segments the lung image. The third module refines the output of SPQT to get the fine segmentation of lungs. Fig. 1 explains the proposed method with detailed explanation of each major step. 


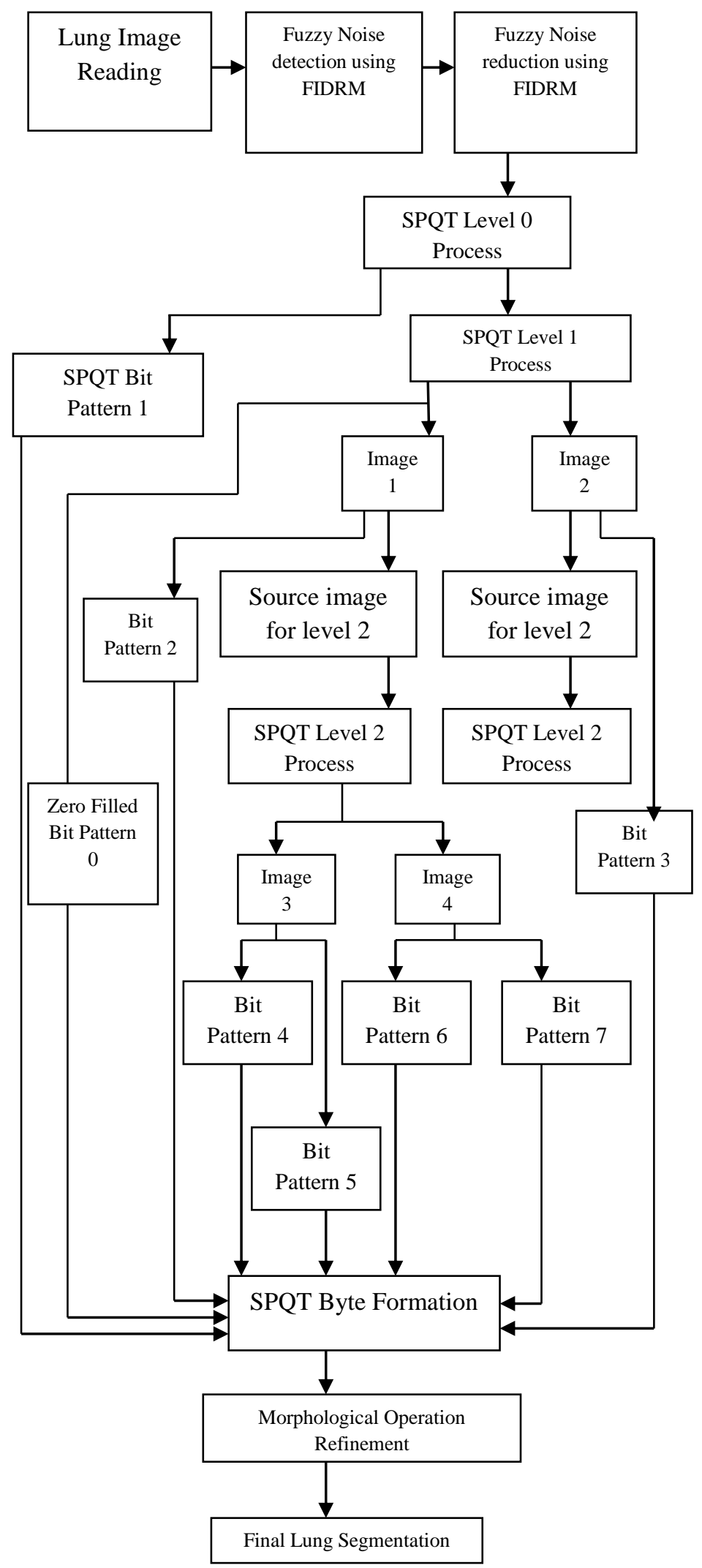

Figure.1 Block diagram of the proposed method 


\begin{tabular}{|c|c|c|}
\hline NW & N & NE \\
\hline W & $(i, j)$ & E \\
\hline SW & S & SE \\
\hline
\end{tabular}

Figure. 2 Demonstration of $3 \times 3$ window

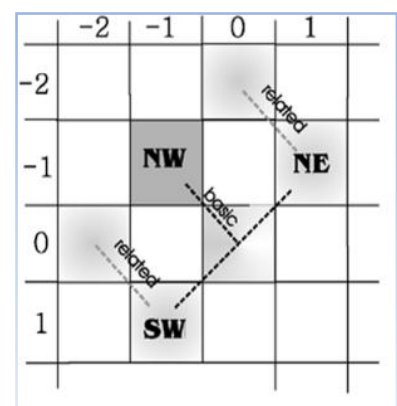

Figure.3 Illustration of relative gradient

\subsection{A FIDRM based noise reduction}

The noise occurrence in lung medical image affects the accuracy of segmentation. A noise reduction scheme can be solved this problem. So this paper uses the 'Fuzzy impulse noise detection and reduction method' (FIDRM) to generate noise free image. The FIDRM noise detection is performed by a $3 \times 3$ window which is illustrated in Fig. 2.

If we denote $A$ as the input lung image, then the basic gradient $\nabla$ can be defined as

$$
\begin{gathered}
\nabla=A(i+k, j+l)-A(i, j) \\
k \in[-1,0, l] \quad l \in[-1,0,1]
\end{gathered}
$$

The related gradient can be found based on Fig. 3 and Eq. (2).

The membership functions SMALL, LARGE, BIG-NEGATIVE and BIG-POSITIVE are derived and computed based on the paper [44].The fuzzy rules constructed in the paper [44] are considered to find the $\nabla_{R}^{F} A(i, j)$ which is the representative of the rate of noisiness. The fuzzy based noise detection is proceeded using the following fuzzy rule.

If most of the eight $\nabla_{R}^{F} A(i, j)$ are larger then End

$A(i, j)$ is an impulse noise pixel

The noise reduction process is gone through the layer based window (multiple order elements) and an iterative procedure is applied for each order of multiple order window elements using a mean based approach.

\subsection{SPQT based partial lung segmentation}

The binary tree processing approach is regarded here for implementing SPQT lung segmentation. The noise free image is used as input and the histogram Hist(i) is computed using Eq.(2).

$$
\begin{aligned}
& \text { Hist }(i)=\text { InputImageIntensity Count }(i) \\
& \text { Where, } \mathrm{i}=[0,255]
\end{aligned}
$$

The histogram is often being with speckled type variations and to remove that, a smoothing operation is worked out on the histogram surface. Then the highest peak in between 32 to 192 ranges is determined by Eq. (3).

Peak=PeakDetection $($ SmoothOperation $($ Hist $(32: 192)))$

Where, peak - computed peak value,

Peak detection ( ) - peak detection function, Smooth Operation ( ) - Histogram smoothing function.

The range 32 to 192 indicates the most probable range of lung part in medical image. The noise free image is quantized by the peak value threshold and as a result two binary images are generated. Among those two binary images, one is filled to bit pattern value 1 . The second image is refilled by the intensity values of original noise free image for the pixels which are higher value than the computed peak value. This output is known as level 1 operation and from this operation only one pattern is constructed.

The next level (second level) of processing two intensity images is generated with the help of peak detection. The Image 1 generates a couple of results and the first image is shifted as bit pattern 2 and the next is taken as the input of next iteration. The Image 2 constructs the bit pattern 3 and another image to be a source of next iteration. So, now three bit patterns are formed.

The third iteration creates bit patterns 4 , bit patterns 5 , bit patterns 6 and bit patterns 7 . The unknown bit pattern is still not created and to form that, an image with entire zeros is assigned as bit pattern 0 . These eight bit patterns are subsequently pile up in horizontal order using Eq. (4).

$$
\begin{aligned}
& I_{S P Q T}^{i, j}=I_{B P 0}^{i, j} \times 2^{7}+I_{B P 1}^{i, j} \times 2^{6}+I_{B P 2}^{i, j} \times 2^{5}+I_{B P 3}^{i, j} \times 2^{4} \\
& +I_{B P 4}^{i, j} \times 2^{3}+I_{B P 5}^{i, j} \times 2^{2}+I_{B P 6}^{i, j} \times 2^{1}+I_{B P 7}^{i, j} \times 2^{0}(4)
\end{aligned}
$$

Where, $\quad \mathrm{I}_{\mathrm{SPQT}}^{\mathrm{i}, \mathrm{j}}-$ Byte data by SPQT, $\mathrm{I}_{\mathrm{BPO}}^{\mathrm{i}, \mathrm{j}}-$ Bit 


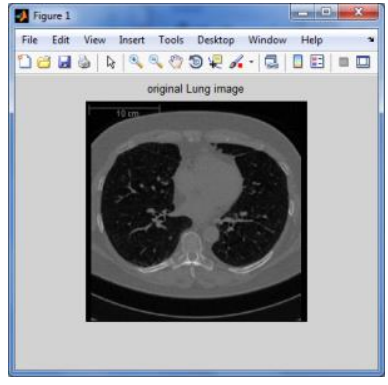

(a)

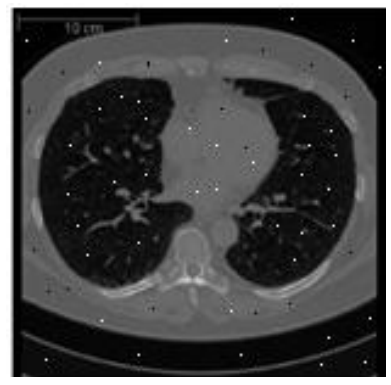

(b)

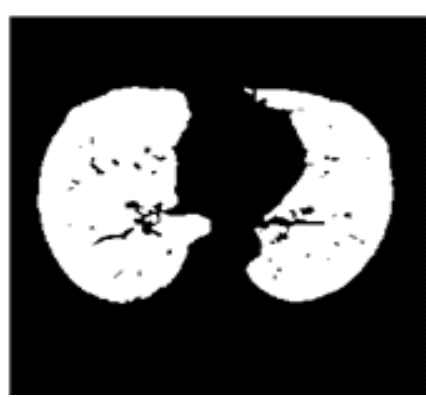

(c)

Figure.4 Output of proposed lung segmentation: (a) Original image, (b) FIDRM noise reduction, and (c) SPQT and morphology based lung segmentation.

Table 1. Peak signal to noise ratio analysis

\begin{tabular}{|l|l|l|l|}
\hline $\begin{array}{l}\text { Lung } \\
\text { Image } \\
\text { Database }\end{array}$ & $\begin{array}{l}\text { Lung } \\
\text { Image }\end{array}$ & $\begin{array}{l}\text { Segmentation } \\
\text { Methods }\end{array}$ & $\begin{array}{l}\text { PSNR (in } \\
\text { db) }\end{array}$ \\
\hline LOLA II & Lola - 1 & Stelios [42] & 62.23 \\
\cline { 3 - 4 } & & Ehsan [43] & 64.13 \\
\cline { 3 - 4 } & Proposed Method & 66.27 \\
\cline { 3 - 4 } & Lola - 2 & Stelios [42] & 62.41 \\
\cline { 3 - 4 } & & Ehsan [43] & 63.99 \\
\cline { 3 - 4 } & Proposed Method & 66.61 \\
\cline { 3 - 4 } & \multirow{5}{*}{ Lola - 3 } & Stelios [42] & 60.72 \\
\cline { 3 - 4 } & & Ehsan [43] & 61.91 \\
\cline { 3 - 4 } & Lola - 4 & Proposed Method & 64.27 \\
\hline & Stelios [42] & 62.27 \\
& Ehsan [43] & 63.91 \\
\cline { 3 - 4 } & Proposed Method & 65.95 \\
\cline { 3 - 4 } & Lola - 5 & Stelios [42] & 61.55 \\
\cline { 3 - 4 } & & Ehsan [43] & 63.71 \\
\cline { 3 - 4 } & Proposed Method & 66.3 \\
\hline
\end{tabular}

pattern0, I I $\mathrm{I}_{\mathrm{BP} 1}^{\mathrm{i} j}-$ Bit pattern1, $\mathrm{I}_{\mathrm{BP} 2}^{\mathrm{i}, \mathrm{j}}-$ Bit pattern2, $\mathrm{I}_{\mathrm{BP} 3}^{\mathrm{i}, \mathrm{j}}-$ Bit pattern3, $\mathrm{I}_{\mathrm{BP} 4}^{\mathrm{i}, \mathrm{j}}-$ Bit pattern4, $\mathrm{I}_{\mathrm{BP} 5}^{\mathrm{i}, \mathrm{j}}-\mathrm{Bit}$ pattern5, $\mathrm{I}_{\mathrm{BP} 6}^{\mathrm{i} j}-$ Bit pattern6, $\mathrm{I}_{\mathrm{BP7}}^{\mathrm{i}, \mathrm{j}}-$ Bit pattern7.

The binarization process generates the intermediate lung segmentation output. The partial result is refined by morphological operations using filling process. The two parameters such as morphological output and the intermediate segmentation results are jointly processed to reach the segmented lung image.

\section{Simulation results}

The new method of lung segmentation separates the left and right lungs from the background of chest CT images. This algorithm can segment lung parts even from the noisy environment. The Lola11 database [41] is used to simulate and test this algorithm to analyze the performance measurements. In Lola11 database 100 images are taken to test the lung segmentation activity. The proposed method is compared against existing version with two papers the Stelios method [42] and the Ehsan method [43] to accomplish the analysis part.

Fig. 4 explains and illustrates about the segmentation output of the proposed method which involves both FIDRM and SPQT. The fig 4(a) shows the original lung image which is to be used for simulation and the fig 4(b) shows the noise free image which is obtained from the FIDRM filter. The fig 4(c) shows the segmented lungs including both left and right lungs with the help of the proposed method.

Table 1 and Fig. 5 illustrate the performance of the proposed method against the existing versions in terms of $\mathrm{db}$. The table 1 contains higher PSNR values for proposed method and lower PSNR values for the existing methods. The higher PSNR values expresses better segmentation and vice versa. So it is proved that the proposed method is the better method.

Table 2 and Fig. 6 analyze the accuracy performance measurement parameter which shows that the proposed method gains higher accuracy whereas the existing methods gains lower accuracy. This is the strong proof for the esteemed performance of the proposed method.

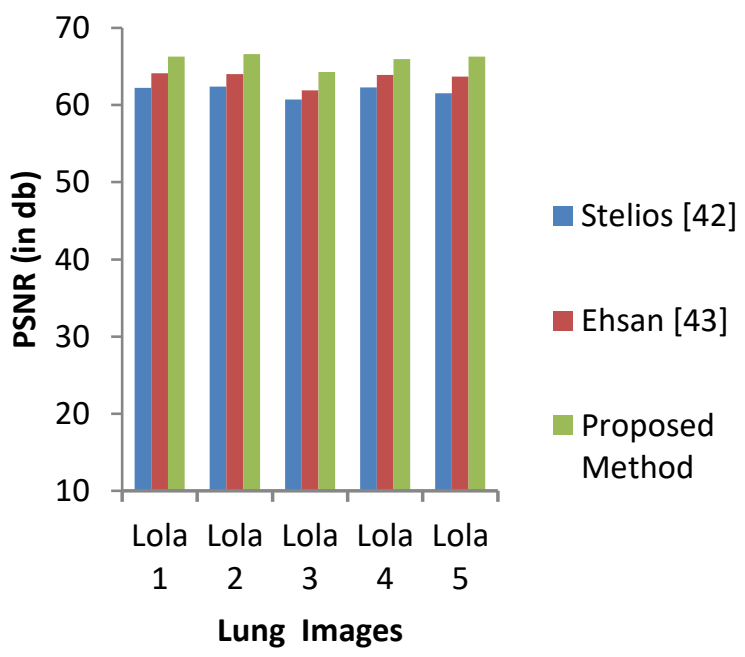

Figure.5 PSNR analysis graph 


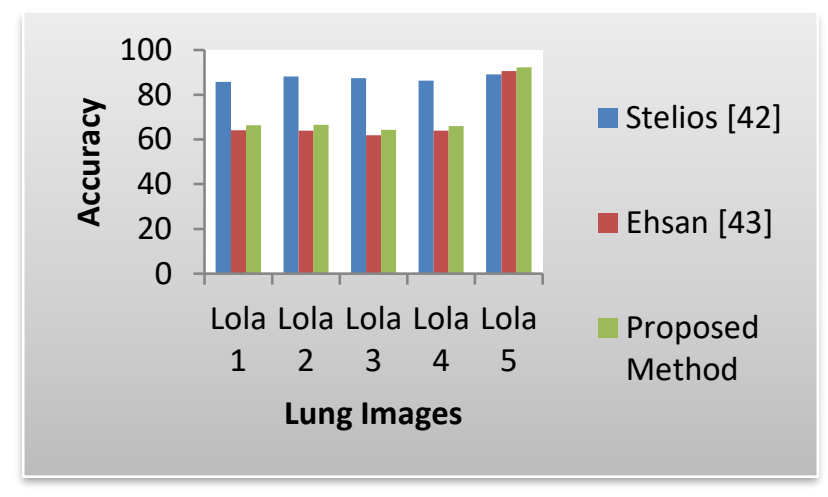

Figure.6 Accuracy analysis chart

Table 2. Accuracy analysis for lung segmentation

\begin{tabular}{|c|c|c|c|}
\hline $\begin{array}{c}\text { Lung } \\
\text { Image } \\
\text { Data } \\
\text { base }\end{array}$ & $\begin{array}{l}\text { Lung } \\
\text { Image }\end{array}$ & $\begin{array}{l}\text { Segmentation } \\
\text { Methods }\end{array}$ & Accuracy \\
\hline \multirow{15}{*}{$\begin{array}{c}\text { LOLA } \\
\text { II }\end{array}$} & \multirow[t]{3}{*}{ Lola - 1} & Stelios [42] & 85.81 \\
\hline & & Ehsan [43] & 88.84 \\
\hline & & Proposed Method & 91.54 \\
\hline & \multirow[t]{3}{*}{ Lola - 2} & Stelios [42] & 88.19 \\
\hline & & Ehsan [43] & 90.43 \\
\hline & & Proposed Method & 92.29 \\
\hline & \multirow[t]{3}{*}{ Lola - 3} & Stelios [42] & 87.35 \\
\hline & & Ehsan [43] & 90.13 \\
\hline & & Proposed Method & 91.21 \\
\hline & \multirow[t]{3}{*}{ Lola - 4} & Stelios [42] & 86.35 \\
\hline & & Ehsan [43] & 88.37 \\
\hline & & Proposed Method & 90.61 \\
\hline & \multirow[t]{3}{*}{ Lola - 5} & Stelios [42] & 89.13 \\
\hline & & Ehsan [43] & 90.61 \\
\hline & & Proposed Method & 92.3 \\
\hline
\end{tabular}

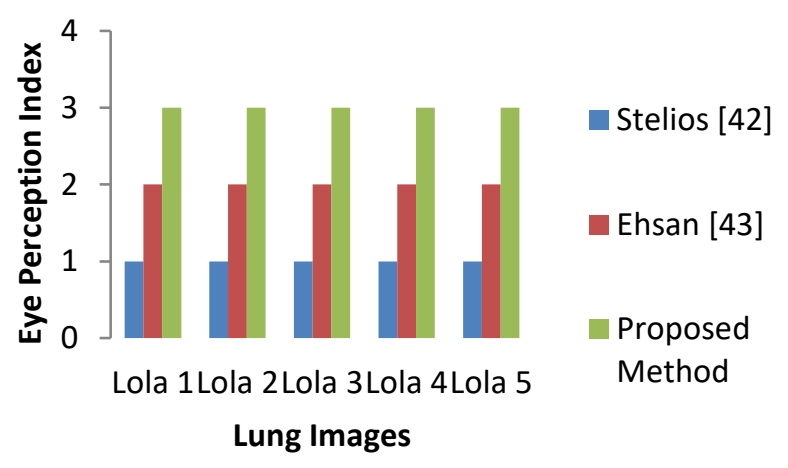

Figure.7 The eye perception index analysis

Table 3 and Fig.7 illustrate the eye perception index based analysis. The eye perception index is computed using the perception behavior of human
Table 3. Eye perception quality analysis

\begin{tabular}{|c|c|c|c|}
\hline $\begin{array}{l}\text { Lung } \\
\text { Image } \\
\text { Database }\end{array}$ & $\begin{array}{l}\text { Lung } \\
\text { Image }\end{array}$ & $\begin{array}{l}\text { Segmentation } \\
\text { Methods }\end{array}$ & $\begin{array}{l}\text { Eye } \\
\text { Percepti } \\
\text { on Index }\end{array}$ \\
\hline \multirow{15}{*}{$\begin{array}{l}\text { LOLA } \\
11\end{array}$} & \multirow[t]{3}{*}{ Lola - 1} & Stelios [42] & 1 \\
\hline & & Ehsan [43] & 2 \\
\hline & & Proposed Method & 3 \\
\hline & \multirow[t]{3}{*}{ Lola - 2} & Stelios [42] & 1 \\
\hline & & Ehsan [43] & 2 \\
\hline & & Proposed Method & 3 \\
\hline & \multirow[t]{3}{*}{ Lola - 3} & Stelios [42] & 1 \\
\hline & & Ehsan [43] & 2 \\
\hline & & Proposed Method & 3 \\
\hline & \multirow[t]{3}{*}{ Lola - 4} & Stelios [42] & 1 \\
\hline & & Ehsan [43] & 2 \\
\hline & & Proposed Method & 3 \\
\hline & \multirow[t]{3}{*}{ Lola - 5} & Stelios [42] & 1 \\
\hline & & Ehsan [43] & 2 \\
\hline & & Proposed Method & 3 \\
\hline
\end{tabular}

beings in the case of output verification in lung segmentation. If the eye perception index is higher than the performance rating by human beings are high for that method so that it can be noticed that the proposed method is best because of its higher index value. The Ehsan [43] method reaches second rank based on this analysis. The proposed method works with the foundation of peak based quantization to obtain the segmented image. The noise reduction using FIDRM filter improves the probability of segmentation. The Successive Peak Quantization Transform method produces the bit patterns as a single byte of information and refined by the morphological operators. This proposed method is a well suitable method to fit with the lung scan hardware. Also, the difficulties existing with the other methods like the severely affected lung images are segmented with the proposed method. This will make pathologist to take strong and correct decision before the surgery.

\section{Conclusion}

The proposed method releases a solution for the lung segmentation issues which are not solved by the existing versions. The complexity with other methods are rectified by this transform. The proposed method provides higher PSNR than the existing methods and it achieves the value as 66.61. The accuracy based algorithm proves that the proposed method is the pre-eminence method over the recent developments of lung segmentation with the numerical value 92.30. The proposed method takes only reasonable time taken which is the most required property of an essential algorithm. So it is concluded that the proposed method is the esteemed 
method than the existing versions in lung segmentation. The fissure enhancement and lung cancer detection modules can be further attached with this method to get more automatic versions of software for pulmonary disease diagnosis.

\section{References}

[1] A. C. Cilva, P. Cezar, and M. Gattas, "Diagnosis of Lung Nodule using Gini Coefficient and skeletonization in CT images", ACM symposium on Applied Computing, 2004.

[2] Y. Lee, T. Hara, H. Fujita, S. Itoh, and T. Ishigaki, "Automated detection of pulmonary nodules in helical CT images based on an improved template matching technique", IEEE Transactions on Medical Imaging, Vol. 20, No.7, pp.595-604, 2001.

[3] S.G. Armato, M.L. Giger, C.J. Moran, J.T. Blackburn, K. Doi, and H. MacMohan, "Computerized detection of pulmonary nodules on CT scans", Radiographs, Vol. 19, No.3, pp.1303-1311, 1999.

[4] K. Doi, H. Macmohan, and S. Katsuragawa, "Computer aided diagnosis in radiology: potential and pitfalls", European Journal of Radiology, Vol.31, pp.97-109, 1997.

[5] J. Wei and G. Li, "Automated Lung Segmentation and Image Quality Assessment for Clinical 3-D/4-D Computed Tomography", IEEE Journal of Translational Engineering in Health and Medicine, Vol. 2, 2014.

[6] A.V. Oppenhaim and R. W. Schafer, DiscreteTime Signal Processing, 3rd ed. Englewood Cliffs, NJ, USA: Prentice-Hall, 2009.

[7] K.P. Murphy, Machine Learning: A Probabilistic Perspective. Cambridge, MA, USA: MIT Press, 2012.

[8] G. Li, "Rapid estimation of 4DCT motionartifact severity based on 1D breathingsurrogate periodicity", Med. Phys., Vol. 41, No. 11, p. 111717, 2014.

[9] J. Wei, A. Yuan, and G. Li, "An automatic toolkit for efficient and robust analysis of 4D respiratory motion", In: Proc. of AAPM, Vol. 41, No.6, 2014.

[10] H. Han, L. Li, F. Han, B. Song, W. Moore, and Z. Liang, "Fast and Adaptive Detection of Pulmonary Nodules in Thoracic CT Images Using a Hierarchical Vector Quantization Scheme", IEEE Journal of Biomedical and Health Informatics, Vol. 19, No. 2, pp. 648-659, 2014.

[11] D. Chen, Z. Liang, M. R. Wax, L. Li, B. Li, and A. E. Kaufman, "A novel approach to extract colon lumen from CT images for virtual colonoscopy", IEEE Transactions on Medical Imaging, Vol. 19, No. 12, pp. 1220-1226, 2000.

[12] L. Li, D. Chen, H. Lu, and Z. Liang, "Segmentation of brain MR images: a selfadaptive online vector quantization approach", In: Proc. of SPIE, Vol. 4322, pp. 1431-1438, 2001.

[13] L. Li, D. Chen, H. Lu, and Z. Liang, "Comparison of quadratic and linear discriminate analyses in the self-adaptive feature vector quantization scheme for MR image segmentation", In: Proc. of Int. Soc. Mag. Reson. Med., Vol. 9, pp. 809, 2001.

[14] F. Han, H. Wang, B. Song, G. Zhang, H. Lu, W. Moore, H. Zhao, and Z. Liang, "A new 3D texture feature based computer-aided diagnosis approach to differentiate pulmonary nodules", In: Proc. of SPIE, Vol. 8670, pp. 86702Z-1-7, 2013.

[15] W. Ju, D. Xiang, B. Zhang, L. Wang, I. Kopriva, and X. Chen, "Random Walk and Graph Cut for Co-Segmentation of Lung Tumor on PET-CT Images", IEEE Transactions on Image Processing, No.12, vol.24, pp. 5854-5867, 2015.

[16] Q. Song, J. Bai, D. Han, S. Bhatia, W. Sun, W. Rockey, and $\mathrm{X}$. Wu, "Optimal co-segmentation of tumor in PET-CT images with context information", IEEE Transactions on Medical Imaging, Vol.32, No.9, pp.1685-1697, 2013.

[17] Y. Boykov and M. P. Jolly, "Interactive graph cuts for optimal boundary \& region segmentation of objects in ND images", IEEE International Conference on Computer Vision, pp. 105-112, 2001.

[18] Y. Boykov and O. Veksler, "Graph Cuts in Vision and Graphics: Theories and Applications", Handbook of Mathematical Models in Computer Vision, Springer, 2006.

[19] Y. Boykov and V. Kolomogorov, "An Experimental Comparison of Min-Cut/MaxFlow Algorithms for Energy Minimization in Vision", IEEE Transactions on Pattern Analysis and Machine Intelligence, Vol. 28, No. 9, pp. 1124-1137, 2004.

[20] Y. Boykov, O. Veksler, and R. Zabih, "Fast Approximate Energy Minimization via Graph Cuts", IEEE Transactions on Pattern Analysis and Machine Intelligence, Vol. 23, No.11, pp. 1222-1239, 2001.

[21] Y. Boykov and G. Funka-Lea, "Graph cuts and efficient N-D image segmentation", International Journal of Computer Vision, Vol. 70, No. 2, pp. 109-131, 2006. 
[22] A. Soliman, F. Khalifa, A. Elnakib, M. Abou El-Ghar, N. Dunlap, B. Wang, G. Gimelfarb, R. Keynton, and A. El-Baz, "Accurate Lungs Segmentation on CT Chest Images by Adaptive Appearance-Guided Shape Modeling", IEEE Transactions on Medical Imaging, No. 1, Vol. 36, pp. 263-276, 2017.

[23] B. Glocker, A. Sotiras, N. Komodakis, and N. Paragios, "Deformable medical image registration: Setting the state of the art with discrete methods", Ann. Rev. Biomedical Eng., Vol. 13, pp. 219-244, 2011.

[24] T. Lindeberg, "Generalized Gaussian scalespace axiomatics comprising linear scale-space, affine scale-space and spatio-temporal scalespace", Journal of Mathematical. Imaging Vis., Vol. 40, No. 1, pp. 36-81, 2011.

[25] E. Cavalcanti Neto, P.C. Cortez, T.S. Cavalcante, V.E. Rodrigues, P.P. Rebouças Filho, and M.A. Holanda, "3D Lung Fissure Segmentation in TC images Based in Textures", IEEE Latin America Transactions, Vol. 14, No. 1, pp. 254-258, 2016.

[26] J. Pu, J.K. Leader, B. Zheng, F. Knollmann, C. Fuhrman, F.C. Sciurba, and D. Gur, "A computational geometry approach to automated pulmonary fissure Segmentation in CT examinations", IEEE Transactions on Medical Imaging, Vol. 28, No. 5, pp. 710-719, 2009.

[27] M. Anthimopoulos, S. Christodoulidis, L. Ebner, A. Christe, and S. Mougiakakou, "Lung Pattern Classification for Interstitial Lung Diseases Using a Deep Convolutional Neural Network", IEEE Transactions on Medical Imaging, Vol. 35, No. 5, pp. 1207-1216, 2016.

[28] K. R. Heitmann, H. Kauczor, P. Mildenberger, T. Uthmann, J. Perl, and M. Thelen, "Automatic detection of ground glass opacities on lung HRCT using multiple neural networks", Eur. Radiol., Vol. 7, No. 9, pp. 1463- 1472, 1997.

[29] S. Delorme, M.A. Keller-Reichenbecher, I. Zuna, W. Schlegel, and G. V. Kaick, "Usual interstitial pneumonia: quantitative assessment of high-resolution computed tomography findings by computer-assisted texture-based image analysis", Investigative radiology, Vol. 32, No. 9, pp. 566-574, 1997.

[30] C. Xiao, Berend C. Stoel, M. Els Bakker, Y. Peng, Jan Stolk, and M. Staring, "Pulmonary Fissure Detection in CT Images Using a Derivative of Stick Filter", IEEE Transactions on Medical Imaging, Vol. 35, No. 6, pp. 14881500, 2016.
[31] J. Pu, Z. Wang, S. Gu, C. Fuhrman, J. K. Leader, X. Meng, J. Tedrow, and F.C. Sciurba, "Pulmonary fissure integrity and collateral ventilation in copd patients", PLOS ONE, Vol. 9, No. 5, p. e96631, 2014.

[32] Arnaud A. A. Setio, F. Ciompi, G. Litjens, P. Gerke, C. Jacobs, Sarah J. Van Riel, M. Marie W. Wille, M. Naqibullah, Clara I. Sanchez, B. V. Ginneken, "Pulmonary nodule detection in CT images: false positive reduction using multi-view convolutional Networks", IEEE Transactions on Medical Imaging, Vol. 35, No. 5, pp. 1160-1169, 2016.

[33] K. Murphy, B. V. Ginneken, A.M.R. Schilham, B. J. de Hoop, H.A. Gietema, and M. Prokop, "A large scale evaluation of automatic pulmonary nodule detection in chest CT using local image features and k-nearest-neighbour classification", Medical Image Analysis, Vol. 13, pp. 757-770, 2009.

[34] C. Jacobs, E. M. van Rikxoort, T. Twellmann, E. T. Scholten, P. A. de Jong, J. M. Kuhnigk, M. Oudkerk, H. J. de Koning, M. Prokop, C. Schaefer-Prokop, and B. van Ginneken, "Automatic detection of subsolid pulmonary nodules in thoracic computed tomography images", Medical Image Analysis, Vol. 18, pp. 374-384, 2014.

[35] A. A. A. Setio, C. Jacobs, J. Gelderblom, and B. van Ginneken, "Automatic detection of large pulmonary solid nodules in thoracic CT images," Medical Physics, Vol. 42, No. 10, pp. 56425653, 2015.

[36] S. Zhou, Y. Cheng, and S. Tamura, "Automated lung segmentation and smoothing techniques for inclusion of juxtapleural nodules and pulmonary vessels on chest CT images", Biomedical Signal Processing and Control, Vol. 13, pp. 62-70, 2014.

[37] E.G. Chan, J.R. Landreneau, M.J. Schuchert, D.D. Odell, S. Gu, J. Pu, J.D. Luketich, and R.J. Landreneau, "Pre-Operative (3-D) CT Lung Reconstruction Prior to Anatomic Segmentectomy or Lobectomy for Stage I", The Journal of Thoracic and Cardiovascular Surgery, Vol. 150, No. 3, pp. 523-8, 2015.

[38] X. Song, M. Cheng, B. Wang, S. Huang, and X. Huang, "Preoperative Planning for Liver Surgery based on CT Images", In: Proc. of Engineering, Vol. 24, pp. 133-137, 2011.

[39] E.E. Nithila and S.S. Kumar, "Segmentation of lung nodule in CT data using active contour model and Fuzzy C-mean clustering", Alexandria Eng. J., Vol.55, No. 3, pp. 25832588, 2016. 
[40] K. Zhang, L. Zhang, H. Song, and W. Zhou, "Active contours with selective local or global segmentation: a new formulation and level set method", Image Vis. Comput., Vol. 28, pp. 668-676, 2010.

[41] https:// lola11. grand - challenge. Org / details/

[42] S. Krinidis and V. Chatzis, "A robust fuzzy local information Cmeans Clustering Algorithm", IEEE Transactions on Image Processing, Vol. 19, No.5, pp. 1328-1337, 2010.

[43] Ehsan Hosseini - Asl, Jacek M Zurada, G. Gimelfarb and A. El - Baz, "3D lung segmentation Based on Incremental constrained non negative matrix Factorization", IEEE Transaction on Biomedical Engineering, Vol. 63, No. 5, pp. $952-963,2015$.

[44] S. Schulte, M. Nachtegael, V. D. Witte, D. V. Der Weken, and Etienne E. Kerre, "A fuzzy impulse noise detection and reduction method", IEEE Transactions on Image Processing, Vol. 15, No.5, pp. 1153-1162, 2006. 